\title{
The Analysis of Decomposition Methods for Support Vector Machines
}

\author{
Chih-Chung Chang, Chih-Wei Hsu, and Chih-Jen Lin
}

\begin{abstract}
The support vector machine (SVM) is a new and promising technique for pattern recognition. It requires the solution of a large dense quadratic programming problem. Traditional optimization methods cannot be directly applied due to memory restrictions. Up to now, very few methods can handle the memory problem and an important one is the "decomposition method." However, there is no convergence proof so far. In this paper, we connect this method to projected gradient methods and provide theoretical proofs for a version of decomposition methods. An extension to bound-constrained formulation of SVM is also provided. We then show that this convergence proof is valid for general decomposition methods if their working set selection meets a simple requirement.
\end{abstract}

Index Terms-Decomposition methods, projected gradients, support vector machines.

\section{INTRODUCTION}

The support vector machine (SVM) is a new and very promising classification technique for pattern recognition. Surveys of SVM are, for example, Burges [1], Cortes and Vapnik [2], Schölkopf et al. [3], and Vapnik [4]. Giving training vectors $v_{i}, i=1, \cdots, m$ of length $k$, and a vector $a$ defined as follows:

$$
a_{i}=\left\{\begin{aligned}
1, & \text { if } v_{i} \text { in class } 1, \\
-1, & \text { if } v_{i} \text { in class } 2
\end{aligned}\right.
$$

the support vector technique in general requires the solution of the following quadratic programming problem:

$$
\begin{aligned}
\min & \frac{1}{2} x^{T} Q x-e^{T} x \\
& a^{T} x=0, \\
& 0 \leq x_{i} \leq C, \quad i=1, \cdots, n
\end{aligned}
$$

where $e$ is the vector of all ones, $C$ is the upper bound of all variables, $Q$ is a positive semidefinite matrix. Possible choices of $Q_{i j}$ are, for example, $a_{i} a_{j}\left(\left(v_{i}^{T} v_{j}\right) / k\right)^{d}$ and $a_{i} a_{j} e^{-\left\|v_{i}-v_{j}\right\|^{2} / k}$. Note that $v_{i}$ is considered as a support vector if $x$ is the solution of (1) and $x_{i}>0$.

The difficulty of solving (1) is the density of $Q$ because $Q_{i j}$ is in general not zero and $Q$ becomes a fully dense matrix. Hence a prohibitive amount of memory is required to store the matrix. Thus, traditional optimization algorithms such as Newton, quasi-Newton, etc., cannot be directly applied. Several authors (e.g., [5]-[10]) have proposed methods with successful

Manuscript received November 10, 1999; revised April 18, 2000. This work was supported in part by the National Science Council of Taiwan, R.O.C., under Grant NSC-88-2213-E-002-097.

The authors are with the Department of Computer Science and Information Engineering, National Taiwan University, Taipei 106, Taiwan, R.O.C., (e-mail cjlin@csie.ntu.edu.tw).

Publisher Item Identifier S 1045-9227(00)05952-X. implementations to conquer this difficulty. Unlike the Newton method which usually involves the whole Hessian matrix $Q$, these methods only calculate components of $Q$ when they are required in the current iteration. Among them, an important one is the "decomposition method" proposed by Osuna et al. [8]. As a variation of the active set methods, they separated the index $\{1, \cdots, n\}$ of the training set to two sets $B$ and $N$, where $x_{i}=0$, for $i \in N$ and $B$ is the working set if $x$ is the current iterate of the algorithm. If we denote $x_{B}, x_{N}, e_{B}$, and $e_{N}$ as vectors containing corresponding elements, the objective value is equal to $(1 / 2) x_{B}^{T} Q_{B B} x_{B}-\left(e_{B}+Q_{B N} x_{N}\right)^{T} x_{B}+$ $(1 / 2) x_{N}^{T} Q_{N N} x_{N}-e_{N}^{T} x_{N}$. At each iteration, $x_{N}$ is fixed and the following subproblem with the variable $x_{B}$ is solved:

$$
\begin{gathered}
\min \frac{1}{2} x_{B}^{T} Q_{B B} x_{B}-\left(e_{B}-Q_{B N} x_{N}\right)^{T} x_{B} \\
0 \leq x_{B} \leq C, \quad a_{B}^{T} x_{B}=-a_{N}^{T} x_{N}
\end{gathered}
$$

where $\left[\begin{array}{ll}Q_{B B} & Q_{B N} \\ Q_{N B} & Q_{N N}\end{array}\right]$ is a permutation of the matrix $Q$ and $q$ is the size of $B$. Elements of $x_{N}$ are kept as zero from the beginning. The Karush-Kuhn-Tucker (KKT) condition of (2) includes $0 \leq x_{B} \leq C, a_{B}^{T} x_{B}=-a_{N}^{T} x_{N}$ of (2) and

$$
\begin{aligned}
Q_{B B} x_{B}+Q_{B N} x_{N}-e_{B} & =\sigma a_{B}+\lambda-\mu, \quad \lambda_{i}\left(x_{B}\right)_{i}=0 \\
\mu_{i}\left(C-\left(x_{B}\right)_{i}\right) & =0, \quad \lambda_{i} \geq 0, \mu_{i} \geq 0 .
\end{aligned}
$$

If there is one $\left(x_{B}\right)_{i}$ satisfying $0<\left(x_{B}\right)_{i}<C$, from (3), $\sigma$ could be easily obtained. Since all components of $x_{N}$ are zero, if $x$ is an optimal solution, $x_{N}$ satisfies the KKT condition of (2): $(Q x)_{i}-1-\sigma a_{i} \geq 0$, for $i \in N$. Therefore, the algorithm moves elements of $N$ which violate the KKT condition of (2) to $B$, and move out zero elements from $B$ to $N$. If the size of $B$ is bigger than the number of support vectors, because no cycle happens, the algorithm stops in finite steps.

The main shortcoming of the above algorithm is that we do not know the number of support vectors a priori. To handle this issue, the same authors [11] proposed to use a small number $q$ as the size of $B$. Furthermore, there is no restriction that $x_{N}$ must only contain zeros. To be more precise, any element in $N$ which violates the KKT condition can enter $B$. Since $q$ is small in general (less than 100), this method never faces memory problem. Good numerical results were reported so several implementations and further improvements are given in [5], [9], [10]. However, even though the strict decrease of the objective function still holds, there is no theoretical proof to show that the sequence converges to an optimal solution. This issue has been circulated in the SVM community for a while (see, for example, the discussion in Smola and Schölkopf [12, Sec. 5.5.2], and Keerthi et al. [13, Sec. 3]), but there is no satisfactory solution so far.

The paper by Joachims [5] has drawn us a lot of attention because of its method of updating $B$ and $N$. Unlike the method by 
Osuna et al., where a more "random" selection is used, Joachims solves the following problem in order to select the working set:

$$
\begin{array}{ll}
\min \nabla f\left(x_{k}\right)^{T} d & \\
a^{T} d=0, & -1 \leq d \leq 1 \\
d_{i} \geq 0, & \text { if }\left(x_{k}\right)_{i}=0 \\
d_{i} \leq 0, & \text { if }\left(x_{k}\right)_{i}=C \\
\left|\left\{d_{i} \mid d_{i} \neq 0\right\}\right|=q &
\end{array}
$$

where

$$
\begin{array}{ll}
x_{k} & k \text { th iterate; } \\
f\left(x_{k}\right) & =(1 / 2) x_{k}^{T} Q x_{k}-e^{T} x_{k} ; \\
\nabla f\left(x_{k}\right) & \text { gradient of } f \text { at } x_{k} .
\end{array}
$$

Note that $\left|\left\{d_{i} \mid d_{i} \neq 0\right\}\right|$ means the number of components of $d$ which are not zero. The constraint (4d) implies that a descent direction involving only $q$ variables is obtained. Then components of $x$ with nonzero $d_{i}$ are included in the working set $B$ which is used to construct the subproblem (2). Note that $d$ is only used for identifying $B$ but not as a search direction. Using the decomposition method with the new technique for selecting the working set $B$, Joachims reported promising numerical results. In this paper, we will demonstrate that a variation of his algorithm theoretically converges.

In Section II, we present a more general algorithm with an analysis on the selection of the working set. Then in Section III, we prove the convergence of this algorithm using the techniques of projected gradients. Section IV extends the convergence proofs to bound-constrained formulations of SVM. In Section V, we explain that our convergence proof is valid for general decomposition methods if their working set selection meets a simple requirement. Finally we provide concluding remarks in Section VI.

A preliminary version of this paper was presented in an earlier workshop [14].

\section{GENERAL AlgORITHM}

To describe the new algorithm, we replace the problem (1) by the following form:

$$
\min f(x), x \in \Omega
$$

where $f(x)$ is a continuously differentiable function from $R^{n}$ to $R$ and the set $\Omega$ is the feasible set of (1)

$$
\Omega \equiv\left\{x \mid a^{T} x=0,0 \leq x_{i} \leq C, i=1, \cdots, n\right\} .
$$

Therefore, (1) is a special case of (5) when $f(x)=$ $(1 / 2) x^{T} Q x-e^{T} x$. In addition, we assume $C$ is a finite positive number so $\Omega$ is a bounded set.

In the following we describe a more general algorithm for solving (5).

Step 1) Let $q$ be a positive integer with $2 \leq q \leq n$. Set $k=1$, choose $\mu_{1}, \mu_{2} \in(0,1), \gamma_{1}, \gamma_{2} \geq 0$, and find an $x_{1}$ as the initial solution.

Step 2) Solve

$$
\begin{aligned}
& \min \nabla f\left(x_{k}\right)^{T} d \\
& x_{k}+d \in \Omega \\
& \left|\left\{d_{i} \mid d_{i} \neq 0\right\}\right| \leq q .
\end{aligned}
$$

Assume $B_{k}$ contains indexes for which $d_{i} \neq 0$.

Step 3) If $d=0$, stop and output $x_{k}$ as an optimal solution.

Otherwise, let

$$
\tilde{\nabla} f\left(x_{k}\right)_{i} \equiv \begin{cases}\nabla f\left(x_{k}\right), & \text { if } i \in B_{k} \\ 0, & \text { otherwise. }\end{cases}
$$

Define

$$
\begin{aligned}
& \Omega_{k} \equiv\left\{x \mid x \in \Omega, x_{i}=\left(x_{k}\right)_{i}, i \notin B_{k}\right\}, \\
& R_{k}^{n} \equiv\left\{x \mid x \in R^{n}, x_{i}=\left(x_{k}\right)_{i}, i \notin B_{k}\right\} .
\end{aligned}
$$

The mapping $P_{k}: R_{k}^{n} \rightarrow \Omega_{k}$ is defined by

$$
P_{k}(x) \equiv \arg \min \left\{|z-x|: z \in \Omega_{k}\right\} .
$$

For $\alpha \geq 0$, define $x_{k}(\alpha) \equiv P_{k}\left(x_{k}-\alpha \tilde{\nabla} f\left(x_{k}\right)\right)$, and select $\alpha_{k}>0$ so that $x_{k}^{C} \equiv P_{k}\left(x_{k}-\alpha_{k} \tilde{\nabla} f\left(x_{k}\right)\right)$

$$
f\left(x_{k}^{C}\right) \leq f\left(x_{k}\right)+\mu_{1} \tilde{\nabla} f\left(x_{k}\right)^{T}\left(x_{k}^{C}-x_{k}\right)
$$

and

$$
\alpha_{k} \geq \gamma_{1} \quad \text { or } \quad \alpha_{k} \geq \gamma_{2} \bar{\alpha}_{k}>0
$$

where $\bar{\alpha}_{k}$ satisfies

$$
f\left(x_{k}\left(\bar{\alpha}_{k}\right)\right)>f\left(x_{k}\right)+\mu_{2} \nabla f\left(x_{k}\right)^{T}\left(x_{k}\left(\bar{\alpha}_{k}\right)-x_{k}\right) .
$$

Step 4) Find a new $x_{k+1}$ such that

$$
\left(x_{k+1}\right)_{i}=\left(x_{k}\right)_{i} \quad \text { if } \quad i \notin B_{k} \quad \text { and } \quad f\left(x_{k+1}\right) \leq f\left(x_{k}^{C}\right) \text {. }
$$

Go to Step 2).

Joachims requires that $\left|\left\{d_{i} \mid d_{i} \neq 0\right\}\right|=q$. This may not be always possible, so we modify the equality to $\mid\left\{d_{i} \mid d_{i} \neq\right.$ $0\} \mid \leq q$. Another difference is that we use $x_{k}+d \in \Omega$ instead of $(4 a)-(4 c)$. Note that $(4 a)-(4 c)$ do not ensure the feasibility $x_{k}+d \in \Omega$ so the solution of (4) may not be a feasible direction. However, we use this property for the convergence proof as will be explained in Section III.

The Step 3) of the algorithm searches for a point $x_{k}^{C}$ by following a "partial" projected gradient direction. We called $\tilde{\nabla} f\left(x_{k}\right)$ a "partial" gradient direction since it contains only components of $\nabla f\left(x_{k}\right)$ which are in the working set of $x_{k}$. For a standard projected gradient method (e.g., Calamai and Moré [15], and Bertsekas [16]), $x_{k}^{C} \equiv P\left(x_{k}-\alpha \nabla f\left(x_{k}\right)\right)$, where $P$ is the projection into $\Omega$, and $x_{k}^{C}$ is referred as the Cauchy point. The sufficient decrease of function values at Cauchy points is guaranteed by conditions (9) and (10) and is used for the convergence proof. Then by requiring $f\left(x_{k+1}\right) \leq f\left(x_{k}^{C}\right)$, we will prove that any limit point of the sequence $\left\{x_{k}\right\}$ is an optimal solution. Note that when $f(x)=(1 / 2) x^{T} Q x-e^{T} x$, $x_{k}^{C}$ in our algorithm is a feasible solution to the subproblem (2). Since most existing decomposition methods obtain the optimal solution of (2) for $x_{k+1}$, in Step 4) the requirement that $f\left(x_{k+1}\right) \leq f\left(x_{k}^{C}\right)$ is satisfied.

Because the use of $\tilde{\nabla} f\left(x_{k}\right)$ instead of $\nabla f\left(x_{k}\right)$ makes a difference in Step 3), there is a new obstacle proving the convergence. In the rest of this section, we will construct the relation between (7) and the following problem:

$$
\begin{array}{r}
\min \nabla f\left(x_{k}\right)^{T} d \\
x_{k}+d \in \Omega .
\end{array}
$$


Definition II.1: A point $x^{*}$ is a stationary point of (5) if $\nabla f\left(x^{*}\right)^{T}\left(x-x^{*}\right) \geq 0$, for all $x \in \Omega$. Immediately, we have the following result.

Lemma II.2: If $O P\left(x_{k}\right)$ is the optimal objective value of (11), $O P\left(x_{k}\right)=0$ if and only if $x_{k}$ is a stationary point.

Proof: If $x_{k}$ is not a stationary point, there is an $x \in \Omega$ such that $\nabla f\left(x_{k}\right)^{T}\left(x-x_{k}\right)<0$. Thus $d=x-x_{k}$ is a feasible solution of (11). The objective value $\nabla f\left(x_{k}\right)^{T} d<0$ causes a contradiction. The proof of the other direction is similar.

We will investigate (11) in detail. Except $a^{T} d=0$, constraints of (11) on $d$ can be written as

$$
l_{i} \leq d_{i} \leq u_{i}, \quad i=1, \cdots, n
$$

where $u_{i} \equiv C-\left(x_{k}\right)_{i} \geq 0, l_{i} \equiv-\left(x_{k}\right)_{i} \leq 0$, and $u_{i} \neq l_{i}$, for all $i$. The KKT condition of (11) is

$$
\begin{aligned}
\nabla f\left(x_{k}\right)-\sigma a & =\lambda-\mu \\
\lambda_{i}\left(d_{i}-l_{i}\right) & =0, \lambda_{i} \geq 0, i=1, \cdots, n \\
\mu_{i}\left(u_{i}-d_{i}\right) & =0, \mu_{i} \geq 0, i=1, \cdots, n
\end{aligned}
$$

where $\sigma$ is a real number, and $\lambda_{i}$ and $\mu_{i}$ are multipliers associated with inequalities. If $d$ is an optimal solution of (11), the property $l_{i} \neq u_{i}, \forall i$ and (12) imply that

$$
\begin{aligned}
d_{i}=l_{i} & \Rightarrow \nabla f\left(x_{k}\right)_{i}-\sigma a_{i}=\lambda_{i} \geq 0, \mu_{i}=0, \\
l_{i}<d_{i}<u_{i} & \Rightarrow \nabla f\left(x_{k}\right)_{i}-\sigma a_{i}=0=\lambda_{i}=\mu_{i}, \\
d_{i}=u_{i} & \Rightarrow \nabla f\left(x_{k}\right)_{i}-\sigma a_{i}=-\mu_{i} \leq 0, \lambda_{i}=0 .
\end{aligned}
$$

As $O P\left(x_{k}\right)$ is the optimal objective value of (11)

$$
\begin{aligned}
O P\left(x_{k}\right)= & \sum_{i=1}^{n} \nabla f\left(x_{k}\right)_{i} d_{i} \\
= & \sum_{i: d_{i}=l_{i}} l_{i}\left(\nabla f\left(x_{k}\right)_{i}-\sigma a_{i}\right) \\
& +\sum_{i: d_{i}=u_{i}} u_{i}\left(\nabla f\left(x_{k}\right)_{i}-\sigma a_{i}\right) .
\end{aligned}
$$

Since each term of (13) is nonpositive, if $j$ is the index such that $d_{i}\left(\nabla f\left(x_{k}\right)_{i}-\sigma a_{i}\right)$ has the smallest value

$$
d_{j}\left(\nabla f\left(x_{k}\right)_{j}-\sigma a_{j}\right) \leq \frac{O P\left(x_{k}\right)}{n} .
$$

Hence if $O P\left(x_{k}\right)<0, d_{j} \neq 0$. As $a_{j} d_{j} \neq 0$, with the constraint $a^{T} d=0$, there are some elements $i$ satisfying

$$
\left(a_{i} d_{i}\right)\left(a_{j} d_{j}\right)<0
$$

Furthermore, since all $a_{i}, i=1, \cdots, n$ are one or -1 , there is at least one index $r$ satisfying (15) such that

$$
\left|d_{r}\right| \geq \frac{\left|d_{j}\right|}{n}
$$

Now we are ready to switch back to (7) by considering the following problem:

$$
\begin{array}{ll}
\min & \nabla f\left(x_{k}\right)^{T} d \\
& x_{k}+d \in \Omega \\
& d_{i}=0, \quad \forall i \neq j \text { and } i \neq r .
\end{array}
$$

If $\left|d_{r}\right| \leq\left|d_{j}\right|$, define $\bar{d}_{j} \equiv\left(\left|d_{r}\right| /\left|d_{j}\right|\right) d_{j}$. Then $a_{j} \bar{d}_{j}+a_{r} d_{r}=0$ and from (13), (14), and (16)

$$
\begin{aligned}
d_{r} & \nabla f\left(x_{k}\right)_{r}+\bar{d}_{j} \nabla f\left(x_{k}\right)_{j} \\
& =d_{r} \nabla f\left(x_{k}\right)_{r}+\frac{\left|d_{r}\right|}{\left|d_{j}\right|} d_{j} \nabla f\left(x_{k}\right)_{j} \\
& =d_{r}\left(\nabla f\left(x_{k}\right)_{r}-\sigma a_{r}\right)+\frac{\left|d_{r}\right|}{\left|d_{j}\right|} d_{j}\left(\nabla f\left(x_{k}\right)_{j}-\sigma a_{j}\right) \\
& \leq \frac{1}{n^{2}} O P\left(x_{k}\right) .
\end{aligned}
$$

A similar setting can be made for the case when $\left|d_{r}\right|>\left|d_{j}\right|$. Therefore, by assigning other elements of $d$ to be zero, we find a feasible solution of (17) with objective value less than $\left(1 / n^{2}\right) O P\left(x_{k}\right)$. As this solution is also a feasible point of (7), if $\tilde{O P}\left(x_{k}\right)$ is the optimal objective value of (7), we have the following lemma.

Lemma II.3: $n^{2} \tilde{O P}\left(x_{k}\right) \leq O P\left(x_{k}\right)$.

From Lemma II.3, the fact that $O P\left(x_{k}\right) \leq \tilde{O P}\left(x_{k}\right)$, and Lemma II.2, we have the following lemma.

Lemma II.4: $\tilde{O P}\left(x_{k}\right)=0$ if and only if $x_{k}$ is a stationary point.

\section{CONVERGENCE PROOF}

Based on the analysis on special structure of SVM formulations in Section II, in this section we will provide the convergence proof.

As the only difference between $P_{k}$ and $P$ is the restriction on the domain variable $x$ such that $x_{i}=\left(x_{k}\right)_{i}, i \notin B_{k}$, following from Calamai and Moré [15], we have the following lemma.

Lemma III.1: Let $P_{k}$ be the projection into $\Omega_{k}$.

1) If $z \in \Omega_{k}$, then

$$
\left(P_{k}(x)-x\right)^{T}\left(z-P_{k}(x)\right) \geq 0, \quad \forall x \in R_{k}^{n} .
$$

2) $P_{k}$ is a monotone operator, that is

$$
\left(P_{k}(y)-P_{k}(x)\right)^{T}(y-x) \geq 0, \quad \forall x, y \in R_{k}^{n} .
$$

If $P_{k}(y) \neq P_{k}(x)$ then strict inequality holds.

3) Given $x \in R_{k}^{n}$ and $d \in R^{n}, d_{i}=0, i \notin B_{k}$, the function $\psi$ defined by

$$
\psi(\alpha) \equiv \frac{\left\|P_{k}(x+\alpha d)-x\right\|}{\alpha}, \quad \alpha>0
$$

is nonincreasing.

We define $x_{k}(\alpha) \equiv P_{k}\left(x_{k}-\alpha \tilde{\nabla} f\left(x_{k}\right)\right)$, a function of $\alpha$, which yields the following inequalities: From (18)

$$
\tilde{\nabla} f\left(x_{k}\right)^{T}\left(x_{k}-x_{k}(\alpha)\right) \geq \frac{\left\|x_{k}(\alpha)-x_{k}\right\|^{2}}{\alpha}, \quad \alpha>0 \text {. }
$$

Therefore

$$
\begin{aligned}
& \left\|\left(x_{k}-\alpha \tilde{\nabla} f\left(x_{k}\right)\right)-x_{k}\right\|\left\|x_{k}(\alpha)-x_{k}\right\| \\
& \quad \geq\left(\left(x_{k}-\alpha \tilde{\nabla} f\left(x_{k}\right)\right)-x_{k}\right)^{T}\left(x_{k}(\alpha)-x_{k}\right) \\
& \quad \geq\left\|x_{k}(\alpha)-x_{k}\right\|^{2}
\end{aligned}
$$

and consequently

$$
\left\|\alpha \tilde{\nabla} f\left(x_{k}\right)\right\| \geq\left\|x_{k}(\alpha)-x_{k}\right\|
$$


From (19), if $\alpha \geq \beta$, then

$$
\tilde{\nabla} f\left(x_{k}\right)^{T}\left(x_{k}-x_{k}(\alpha)\right) \geq \tilde{\nabla} f\left(x_{k}\right)^{T}\left(x_{k}-x_{k}(\beta)\right) .
$$

The following lemma ensures that there is an $x_{k}^{C}$ satisfying the requirements in Step 3 of the algorithm.

Lemma III.2: If $x_{k}$ is not a stationary point of (5), then

1) For all $\alpha>0, \tilde{\nabla} f\left(x_{k}\right)^{T}\left(x_{k}(\alpha)-x_{k}\right)<0$.

2) There is a $\mu$ in $(0,1)$, and an $\hat{\alpha}>0$ such that for all $0<\alpha<\hat{\alpha}$

$$
f\left(x_{k}(\alpha)\right) \leq f\left(x_{k}\right)+\mu \tilde{\nabla} f\left(x_{k}\right)^{T}\left(x_{k}(\alpha)-x_{k}\right)
$$

and

3) There are positive constants $\gamma_{1}$ and $\gamma_{2}$, and constants $\mu_{1}$ and $\mu_{2}$ in $(0,1)$, and $\alpha_{k}>0$ such that (9) and (10) are satisfied.

Proof: If 1) is false, we can find an $\bar{\alpha}$ such that

$$
\tilde{\nabla} f\left(x_{k}\right)^{T}\left(x_{k}(\bar{\alpha})-x_{k}\right) \geq 0 .
$$

Then from (23), for all $0 \leq \alpha \leq \bar{\alpha}, \tilde{\nabla} f\left(x_{k}\right)^{T}\left(x_{k}(\alpha)-x_{k}\right) \geq 0$. However, from (21)

$$
\tilde{\nabla} f\left(x_{k}\right)^{T}\left(x_{k}(\alpha)-x_{k}\right) \leq-\frac{\left\|x_{k}(\alpha)-x_{k}\right\|^{2}}{\alpha} \leq 0 .
$$

Therefore, $x_{k}(\alpha)=x_{k}$, for all $0 \leq \alpha \leq \bar{\alpha}$. Because $x_{k}$ is not a stationary point, from Lemma II.4, (7) has a solution $d^{*} \neq$ 0 and there exists an $\hat{\alpha}<\bar{\alpha}$ such that $\left\|\hat{\alpha} \tilde{\nabla} f\left(x_{k}\right)\right\| \leq\left\|d^{*}\right\|$. Because $\tilde{\nabla} f\left(x_{k}\right)^{T} d^{*}<0$, we can find an $r \neq 0$ such that $\left(-\hat{\alpha} \tilde{\nabla} f\left(x_{k}\right)-\left(d^{*} / r\right)\right)^{T} d^{*}=0$. Then

$$
\left\|\left(x_{k}+\frac{d^{*}}{r}\right)-\left(x_{k}-\hat{\alpha} \tilde{\nabla} f\left(x_{k}\right)\right)\right\|<\left\|\hat{\alpha} \tilde{\nabla} f\left(x_{k}\right)\right\| .
$$

This contradicts the property that $\arg \min \left\{\| z-\left(x_{k}-\right.\right.$ $\left.\left.\hat{\alpha} \tilde{\nabla} f\left(x_{k}\right)\right) \|: z \in \Omega_{k}\right\}=x_{k}$.

For 2), because $x_{k}$ is not a stationary point and $f$ is continuously differentiable, from 1) of this Lemma and (21), we have

$$
\begin{aligned}
\left|\frac{-f\left(x_{k}\right)+f\left(x_{k}(\alpha)\right)}{\tilde{\nabla} f\left(x_{k}\right)^{T}\left(x_{k}(\alpha)-x_{k}\right)}-1\right| & \leq \frac{o\left(\left\|x_{k}(\alpha)-x_{k}\right\|\right)}{-\tilde{\nabla} f\left(x_{k}\right)^{T}\left(x_{k}(\alpha)-x_{k}\right)} \\
& \leq \frac{\alpha o\left(\left\|x_{k}(\alpha)-x_{k}\right\|\right)}{\left\|x_{k}(\alpha)-x_{k}\right\|^{2}} .
\end{aligned}
$$

From (20), $\left\|x_{k}(\alpha)-x_{k}\right\| / \alpha$ is nonincreasing. On the other hand, from (22), $\left\|x_{k}(\alpha)-x_{k}\right\| \leq \alpha\left\|\tilde{\nabla} f\left(x_{k}\right)\right\|$, so

$$
\frac{\alpha}{\left\|x_{k}(\alpha)-x_{k}\right\|}=O(1) \text {. }
$$

Thus, (25) implies

$$
\lim _{\alpha \rightarrow 0^{+}} \frac{-f\left(x_{k}\right)+f\left(x_{k}(\alpha)\right)}{\tilde{\nabla} f\left(x_{k}\right)^{T}\left(x_{k}(\alpha)-x_{k}\right)}=1 .
$$

Hence the result (24) immediately follows.

We prove 3) by giving an example from Bertsekas [17]. Define $\alpha_{k}=\gamma_{2}^{m_{k}} \gamma_{1}$, where $m_{k}$ is the smallest integer such that (24) is satisfied. From 2), we know this $\alpha_{k}$ exists. Then by using this $x_{k}\left(\alpha_{k}\right)$ as $x_{k}^{C}$, both (9) and (10) are satisfied.

The following theorem from Calamai and Moré [15] is very important to our convergence proof. Because we are now using $\tilde{\nabla} f\left(x_{k}\right)$ instead of $\nabla f\left(x_{k}\right)$ as the search direction of the
Cauchy step, to demonstrate its validity, here we present the whole proof.

Theorem III.3: Let $f: R^{n} \rightarrow R$ be continuously differentiable on $\Omega$

$$
\lim _{k \rightarrow \infty} \frac{\left\|x_{k}^{C}-x_{k}\right\|}{\alpha_{k}}=0 .
$$

Proof: Assume there is an infinite subset $K_{0}$ such that

$$
\frac{\left\|x_{k}^{C}-x_{k}\right\|}{\alpha_{k}} \geq \epsilon, \quad k \in K_{0} \text { and } \lim _{k \rightarrow \infty, k \in K_{0}} x_{k}=x^{*} \text {. }
$$

We will prove that this assumption leads to a contradiction. From (27), for all $k \in K_{0}$

$$
\frac{\left\|x_{k}^{C}-x_{k}\right\|^{2}}{\alpha_{k}} \geq \epsilon \max \left\{\epsilon \alpha_{k},\left\|x_{k}^{C}-x_{k}\right\|\right\} .
$$

Since $\Omega$ is bounded and $x_{k} \in \Omega, \forall k,\left\{x_{k}\right\}$ is bounded, $\left\{f\left(x_{k}\right)\right\}_{k \in K_{0}}$ converges, and condition (9) implies $\left\{\tilde{\nabla} f\left(x_{k}\right)^{T}\left(x_{k}-x_{k}^{C}\right)\right\}_{k \in K_{0}} \rightarrow 0$. Hence (21) and (28) show that

$$
\lim _{k \in K_{0}, k \rightarrow \infty} \alpha_{k}=0 \quad \text { and } \lim _{k \in K_{0}, k \rightarrow \infty}\left\|x_{k}^{C}-x_{k}\right\|=0 .
$$

In particular, we have shown that eventually $\alpha_{k}<\gamma_{1}$ and hence $\alpha_{k} \geq \gamma_{2} \bar{\alpha}_{k}$ where $\bar{\alpha}_{k}$ satisfies (10). Now assign $\bar{x}_{k}^{C}=x_{k}\left(\bar{\alpha}_{k}\right)$ and $\beta_{k}=\min \left(\alpha_{k}, \bar{\alpha}_{k}\right)$. With this, (20) implies

$$
\frac{\left\|x_{k}\left(\beta_{k}\right)-x_{k}\right\|^{2}}{\beta_{k}} \geq \beta_{k}\left(\frac{\left\|x_{k}^{C}-x_{k}\right\|}{\alpha_{k}}\right)\left(\frac{\left\|\bar{x}_{k}^{C}-x_{k}\right\|}{\bar{\alpha}_{k}}\right) .
$$

Since $\left\|x_{k}^{C}-x_{k}\right\| \geq \epsilon \alpha_{k}$ and $\alpha_{k} \geq \gamma_{2} \bar{\alpha}_{k}$ for $k \in K_{0}$, we obtain

$$
\frac{\left\|x_{k}\left(\beta_{k}\right)-x_{k}\right\|^{2}}{\beta_{k}} \geq \epsilon \min \left\{1, \gamma_{2}\right\}\left\|\bar{x}_{k}^{C}-x_{k}\right\| .
$$

Together with (21) and (23), (29) implies that for $k \in K_{0}$

$$
\begin{aligned}
& \min \left\{\tilde{\nabla} f\left(x_{k}\right)^{T}\left(x_{k}-x_{k}^{C}\right), \tilde{\nabla} f\left(x_{k}\right)^{T}\left(x_{k}-\bar{x}_{k}^{C}\right)\right\} \\
& \quad \geq \epsilon \min \left\{1, \gamma_{2}\right\}\left\|\bar{x}_{k}^{C}-x_{k}\right\| .
\end{aligned}
$$

We now use (30) to obtain the desired contradiction. Since $\left\{\tilde{\nabla} f\left(x_{k}\right)^{T}\left(x_{k}-x_{k}^{C}\right)\right\}$ converges to zero, (30) implies that $\left\{\left\|\bar{x}_{k}^{C}-x_{k}\right\|\right\}$ converges to zero. Thus the continuity of $\nabla f$, the fact $\nabla f\left(x_{k}\right)^{T}\left(x_{k}-\bar{x}_{k}^{C}\right)=\tilde{\nabla} f\left(x_{k}\right)^{T}\left(x_{k}-\bar{x}_{k}^{C}\right)$, and the boundedness of $\left\{x_{k}\right\}$ show that

$$
\left|f\left(\bar{x}_{k}^{C}\right)-f\left(x_{k}\right)-\tilde{\nabla} f\left(x_{k}\right)^{T}\left(\bar{x}_{k}^{C}-x_{k}\right)\right|=o\left(\left\|\bar{x}_{k}^{C}-x_{k}\right\|\right) .
$$

Therefore, if

$$
\rho_{k}(\alpha)=\frac{f\left(x_{k}\right)-f\left(x_{k}(\alpha)\right)}{\tilde{\nabla} f\left(x_{k}\right)^{T}\left(x_{k}-x_{k}(\alpha)\right)}
$$

then

$$
\left|\rho_{k}(\bar{\alpha})-1\right| \leq \frac{o\left(\left\|\bar{x}_{k}^{C}-x_{k}\right\|\right)}{\tilde{\nabla} f\left(x_{k}\right)^{T}\left(x_{k}-\bar{x}_{k}^{C}\right)} .
$$

Hence (30) establishes that $\rho_{k}(\bar{\alpha})>\mu_{2}$ for all $k \in K_{0}$ sufficiently large. This is the desired contradiction because (10) guarantees that $\rho\left(\bar{\alpha}_{k}\right)<\mu_{2}$.

We are now in a position to prove the main theorem of this paper. 
Theorem III.4: Let $f: R^{n} \rightarrow R$ be continuously differentiable on $\Omega$. Any limit point of $\left\{x_{k}\right\}$ is a stationary point of (5).

Proof: For any $z \in \Omega_{k}$, from (18)

$$
\begin{aligned}
\alpha_{k} \tilde{\nabla} f\left(x_{k}\right)^{T}\left(x_{k}^{C}-z\right) & \leq-\left(x_{k}^{C}-x_{k}\right)^{T}\left(x_{k}^{C}-z\right) \\
& \leq-\left(x_{k}^{C}-x_{k}\right)^{T}\left(x_{k}-z\right) \\
& \leq\left\|x_{k}^{C}-x_{k}\left|\left\|\mid x_{k}-z\right\| .\right.\right.
\end{aligned}
$$

Hence

$$
\begin{aligned}
\tilde{\nabla} f\left(x_{k}\right)^{T}\left(x_{k}-z\right) \leq & \tilde{\nabla} f\left(x_{k}\right)^{T}\left(x_{k}-x_{k}^{C}\right) \\
& +\frac{\left\|x_{k}^{C}-x_{k}\right\|}{\alpha_{k}}\left\|x_{k}-z\right\| .
\end{aligned}
$$

If $d$ is a direction at $x_{k}$ with $x_{k}+d \in \Omega_{k}$, then setting $z=x_{k}+d$ yields

$$
-\tilde{\nabla} f\left(x_{k}\right)^{T} d \leq \tilde{\nabla} f\left(x_{k}\right)^{T}\left(x_{k}-x_{k}^{C}\right)+\frac{\left\|x_{k}^{C}-x_{k}\right\|}{\alpha_{k}}\|d\| .
$$

Since this inequality is true for all such $d$, with Lemma II.3, for any $x \in \Omega$

$$
\begin{aligned}
& \nabla f\left(x_{k}\right)^{T}\left(x_{k}-x\right) \leq-O P\left(x_{k}\right) \leq-n^{2} \tilde{O P}\left(x_{k}\right) \\
& \quad=n^{2} \max _{x_{k}+d \in \Omega_{k}}-\tilde{\nabla} f\left(x_{k}\right)^{T} d \\
& \quad \leq n^{2}\left(\tilde{\nabla} f\left(x_{k}\right)^{T}\left(x_{k}-x_{k}^{C}\right)+\frac{\left\|x_{k}^{C}-x_{k}\right\|}{\alpha_{k}} \max _{x_{k}+d \in \Omega}\|d\|\right) .
\end{aligned}
$$

Theorem III.3 implies that $\left\|x_{k}^{C}-x_{k}\right\| / \alpha_{k}$ converges to zero. Since $\left\{f\left(x_{k}\right)\right\}$ converges, (9) implies that $\tilde{\nabla} f\left(x_{k}\right)^{T}\left(x_{k}-x_{k}^{C}\right)$ converges to zero. Since $f(x)$ is continuously differentiable on $\Omega$ and $\|d\|$ is bounded, if $x^{*}$ is a limit point of a subsequence $\left\{x_{k}\right\}_{k \in K_{0}}$

$$
\lim _{k \in K_{0}, k \rightarrow \infty} \nabla f\left(x_{k}\right)^{T}\left(x_{k}-x\right)=\nabla f\left(x^{*}\right)^{T}\left(x^{*}-x\right) \leq 0 .
$$

Since this inequality is true for all $x \in \Omega$, from Definition II.1, $x^{*}$ is a stationary point of (5).

Since the quadratic program of SVM is convex, Theorem III.4 assures that any limit point of $\left\{x_{k}\right\}$ is a global minimum.

\section{BOUND-CONSTRAINED FORMULATIONS OF SVM}

Recently several authors [7], [18] have proposed different methods for formulating support vector machines. The resulting quadratic program is a bound-constrained problem

$$
\begin{gathered}
\min \frac{1}{2} x^{T} Q x-e^{T} x \\
0 \leq x_{i} \leq C, \quad i=1, \cdots, n .
\end{gathered}
$$

Note that the matrix $Q$ here is not the same as the matrix $Q$ in (1). In this section we would like to demonstrate that if the proposed algorithm in Section II is applied to this bound-constrained problem, the convergence proof can still follow.

If $\tilde{O P}\left(x_{k}\right)$ and $O P\left(x_{k}\right)$ are defined by the same way, now it is much easier to establish a relation between them. Instead of Lemma II.3, we have the following lemma.

Lemma IV.1: $\lceil(n / q)\rceil \tilde{O P}\left(x_{k}\right) \leq O P\left(x_{k}\right)$.

Proof: First we assume $d^{*}$ is an optimal solution of (11). We then divide $\{1, \cdots, n\}$ to $\lceil(n / q)\rceil$ subsets: $I_{1}, \cdots, I_{\lceil n / q\rceil}$. Let $I_{1}=B_{k}$ and we distribute the rest ele- ments to $I_{2}, \cdots, I_{[n / q]}$ such that each subset has no more than $q$ elements. If we define vectors $d^{i}, i=1, \cdots,\lceil(n / q)\rceil$ as

$$
d_{j}^{i}= \begin{cases}d_{j}^{*}, & j \in I_{i} \\ , 0 & \text { otherwise }\end{cases}
$$

then all $d^{i}, i=1, \cdots,\lceil(n / q)\rceil$, are feasible solutions of (7). Thus

$$
\nabla f\left(x_{k}\right)^{T} d^{i} \geq \tilde{O P}\left(x_{k}\right), \quad \forall i=1, \cdots,\left\lceil\frac{n}{q}\right\rceil .
$$

Therefore

$$
\begin{aligned}
O P\left(x_{k}\right) & =\nabla f\left(x_{k}\right)^{T} d^{*} \\
& =\sum_{i=1}^{\lceil n / q\rceil} \nabla f\left(x_{k}\right)^{T} d^{i} \geq\left\lceil\frac{n}{q}\right\rceil \tilde{O P}\left(x_{k}\right) .
\end{aligned}
$$

It can be clearly seen that Lemmas III.1 and III.2 and Theorem III.3 are still true without any modifications. Then in the proof of Theorem III.4, we use Lemma IV.1 to obtain the convergence result.

\section{General Working Set Selections}

From the proofs in Section III and the discussion in Section IV, we realize that if the optimal objective value $\tilde{O P}\left(x_{k}\right)$ of the working set selection subproblem is relatively small enough, the algorithm presented in Section II always converges.

Corollary V.1: Suppose Step 2) of the algorithm in Section II is replaced by another strategy and $B_{k}$ contains indexes of the selected working set. If the optimal objective value $\tilde{O P}\left(x_{k}\right)$ of the following problem:

$$
\begin{array}{ll}
\min & \nabla f\left(x_{k}\right)^{T} d \\
& x_{k}+d \in \Omega \\
& d_{i}=0, \quad \text { if } i \notin B_{k}
\end{array}
$$

satisfies

$$
M \cdot \tilde{O P}\left(x_{k}\right) \leq O P\left(x_{k}\right) \leq 0, \forall k
$$

where $M$ is a positive constant, Theorem III.4 still holds.

This corollary gives us the flexibility of choosing different types of working sets. For example, a strategy can be as follows:

Solve

$$
\begin{aligned}
\min & \nabla f\left(x_{k}\right)^{T} d \\
& x_{k}+d \in \Omega \\
& \left|\left\{d_{i} \mid d_{i} \neq 0\right\}\right| \leq q / 2
\end{aligned}
$$

and define

$$
\begin{gathered}
B_{k} \equiv\left\{i \mid d_{i} \neq 0\right\} \cup\left\{q / 2 \text { indexes with } d_{i}=0\right. \text { selected } \\
\text { from another strategy }\} .
\end{gathered}
$$

If $\hat{O P}\left(x_{k}\right)$ is the optimal objective value of (33), from Lemma II.3, we have

$$
n^{2} \tilde{O P}\left(x_{k}\right) \leq n^{2} \hat{O P}\left(x_{k}\right) \leq O P\left(x_{k}\right) .
$$

Hence the algorithm converges. Such a strategy might be useful in practice as (7) is a steepest-descent type selection which may not be the best choice for quick convergence. 


\section{CONCLUSION}

In this paper we discuss the convergence of decomposition methods for support vector machines. The proof is based on the connection between a more general algorithm and the techniques of projected gradients. We also demonstrate that the proof can be easily applied to general bound-constrained formulations of SVM. Examples of flexible working set selections are also given.

\section{ACKNOWLEDGMENT}

The third author thanks Dr. J. More for bringing him to the subject of support vector machines and some very helpful discussions.

\section{REFERENCES}

[1] C. J. C. Burges, "A tutorial on support vector machines for pattern recognition," Data Mining Knowl. Disc., vol. 2, no. 2, pp. 121-167, 1998.

[2] C. Cortes and V. Vapnik, "Support-vector network," Mach. Learn., vol. 20, pp. 273-297, 1995.

[3] B. Schölkopf, C. J. C. Burges, and A. J. Smola, Eds., Advances in Kernel Methods-Support Vector Learning. Cambridge, MA: MIT Press, 1998.

[4] V. Vapnik, Statistical Learning Theory. New York: Wiley, 1998.

[5] T. Joachims, "Making large-scale SVM learning practical," in Advances in Kernel Methods-Support Vector Learning, B. Schölkopf, C. J. C. Burges, and A. J. Smola, Eds. Cambridge, MA: MIT Press, 1998.

[6] L. Kaufman, "Solving the quadratic programming problem arising in support vector classification," in Advances in Kernel Methods-Support Vector Learning, B. Schölkopf, C. J. C. Burges, and A. J. Smola, Eds. Cambridge, MA: MIT Press, 1999.
[7] O. L. Mangasarian and D. R. Musicant, "Successive overrelaxation for support vector machines," IEEE Trans. Neural Networks, vol. 10, pp. 1032-1037, Sept. 1999.

[8] E. Osuna, R. Freund, and F. Girosi, "Training support vector machines: An application to face detection," in Proc. CVPR '97, 1997.

[9] J. C. Platt, "Fast training of support vector machines using sequential minimal optimization," in Advances in Kernel Methods-Support Vector Learning, B. Schölkopf, Ed. Cambridge, MA: MIT Press, 1998.

[10] C. Saunders, M. O. Stitson, J. Weston, L. Bottou, B. Schölkopf, and A. Smola, "Support vector machine reference manual," Royal Holloway Coll., Univ. London, Egham, U.K., Tech. Rep. CSD-TR-98-03, 1998.

[11] E. Osuna, R. Freund, and F. Girosi, "An improved training algorithm for support vector machines," in Proc. IEEE NNSP'97, 1997.

[12] A. J. Smola and B. Schölkopf, "A tutorial on support vector regression," Royal Holloway College, Univ. London, Egham, U.K., Neuro COLT Tech. Rep. TR-1998-030, 1998.

[13] S. S. Keerthi, S. K. Shevade, C. Bhattacharyya, and K. R. K. Murthy, "Improvements to platt's SMO algorithm for SVM classifier design," Dept. Mech. Prod. Eng., Nat. Univ. Singapore, Tech. Rep., 1999.

[14] C.-C. Chang, C.-W. Hsu, and C.-J. Lin, "The analysis of decomposition methods for support vector machines," in Workshop Support Vector Machines, IJCAI 99, 1999.

[15] P. H. Calamai and J. J. Moré, "Projected gradient methods for linearly constrained problems," Math. Programming, vol. 39, pp. 93-116, 1987.

[16] D. P. Bertsekas, Nonlinear Programming. Belmont, MA: Athena, 1995.

[17] D. P. Bertsekas, "On the Goldstein-Levitin-Polyak gradient projection method," IEEE Trans. Automat. Contr., vol. AC-21, pp. 174-184, 1976.

[18] T.-T. Friess, N. Cristianini, and C. Campbell, "The kernel adatron algorithm: a fast and simple learning procedure for support vector machines," in Proc. 15th Int. Conf. Machine Learning. San Mateo, CA: Morgan Kaufmann, 1998. 